\title{
Low-Risk Non-mucinous Adenocarcinoma of the Appendix: When Is an Appendectomy Enough?
}

\author{
Kevin M. Turner, MD ${ }^{1}$, and Sameer H. Patel, MD, FACS ${ }^{2}$ \\ ${ }^{1}$ Department of Surgery, University of Cincinnati College of Medicine, Cincinnati, OH; ${ }^{2}$ Department of Surgery, Division \\ of Surgical Oncology, University of Cincinnati College of Medicine, Cincinnati, OH
}

Non-metastatic adenocarcinoma of the appendix, often found incidentally after routine appendectomy, is a rare cancer whose subsequent management still is debated. It is classified into two general categories: mucinous and nonmucinous adenocarcinoma of the appendix (NMACA). The behavior of NMACA, considered similar to that of colon adenocarcinoma, exhibits more aggressive growth and has high rates of lymph node (LN) metastasis. ${ }^{1}$

The management of NMACA involves performing a formal right hemicolectomy (RHC), not only to obtain a margin negative resection, but also to achieve optimal lymph node sampling for appropriate staging. ${ }^{1}$ However, not all NMACAs are created equal. ${ }^{2}$

In this timely study, Straker et al. ${ }^{3}$ used the National Cancer Database (NCDB) to identify a low-risk group of NMACAs for which less extensive surgical resection (i.e., appendectomy/partial colectomy [A/PC]) would be appropriate. They found that patients who had $\mathrm{T} 1$ tumors with good to moderate differentiation and no lymphovascular invasion (LVI) had a low risk of LN metastasis (1.8\%). In contrast, positive LNs were found in roughly one fourth of all other patients. Examination of the entire patient cohort showed that RHC was associated with better overall survival (OS) than an A/PC. However, in the small group with a low risk of LN metastasis, survival did not differ between

(C) Society of Surgical Oncology 2021

First Received: 6 December 2021

Accepted: 7 December 2021;

Published Online: 10 January 2022

S. H. Patel, MD, FACS

e-mail: patel5se@ucmail.uc.edu the two operative groups. Their results support performing a less extensive oncologic resection for selected patients with NMACA and a favorable pathology.

The findings from Straker et al. ${ }^{3}$ are not completely surprising. For selected early-stage colon and rectal cancer with disease confined to the mucosa, favorable pathologic characteristics, and a low risk of nodal metastases, local resection is a reasonable treatment option. The same has been observed for well-differentiated appendiceal neuroendocrine tumors, and most recently, for T1 welldifferentiated appendiceal adenocarcinomas, which require only a simple appendectomy. In the recent study, AlMasri et al. ${ }^{2}$ used the NCDB to examine the outcomes for patients who underwent appendectomy versus right colectomy for T1 (both mucinous and non-mucinous) appendix adenocarcinoma. They found that the OS was better when a right colectomy was performed for moderately or poorly differentiated tumors but not for well-differentiated tumors, similar to the findings of this study.

Lymph node status is an important gatekeeper for the colon as well as for appendiceal adenocarcinoma. Positive lymph nodes upstage patients from stage 2 to 3 and qualify them for adjuvant therapy. ${ }^{4}$ In the appropriately staged patient, adjuvant therapy improves prognosis. Therefore, knowing when to perform an RHC for adequate nodal staging is crucial.

In this study, very few patients in the low-risk group received adjuvant therapy, and the impact on survival in this group is not known. However, in another NCDB study examining patients who underwent a right colectomy, adjuvant therapy had an OS benefit only for NMACA stage 3 disease but not for stage 2 disease. ${ }^{5}$ This again highlights the importance of accurately staging patients. 
Despite limitations of the NCDB, it does provide a snapshot into real-world practice patterns. In this study, slightly more than one fourth of the patients underwent an A/PC for non-low-risk disease, who arguably should have received a right colectomy. The data show that less extensive resections are being performed in clinical practice. Straker et al. ${ }^{3}$ not only emphasized the group with a low risk of having nodal metastases; they also identified which patients have almost a $30 \%$ risk of positive lymph nodes (T2 or greater, poorly differentiated, and those with lymphovascular invasion) and therefore should undergo an RHC.

Although the authors performed a detailed analysis in this study, some questions remain unanswered. The risk of local or distant recurrence would be an ideal end point for this study to determine the impact of potentially leaving residual nodal disease behind when an appendectomy is performed. However, the NCDB does not provide recurrence data, and this question may be better answered by granular institutional data. Along those lines, it should be noted that even in a low-risk group, an $\mathrm{A} / \mathrm{PC}$ is not enough. These patients also need to undergo routine surveillance. ${ }^{6}$ If a late recurrence is identified, it is unclear whether these patients can then be salvaged without an impact on OS.

Another caution for clinicians evaluating these data is the sample size. Despite the impressive size of the initial cohort, after the low-risk patients had been selected out, only 37 underwent an A/PC. When additional subset analyses are performed on a smaller group, obtaining meaningful statistical analyses is difficult. However, with the potential exception of multicenter collaboratives, the NCDB is one of the largest databases capable of answering questions for this rare patient population.
We applaud Straker et al. ${ }^{3}$ for conducting a thorough investigation into identification of low-risk patients with NMACA who can forego a more extensive operation. With the challenges of performing randomized clinical trials for such a rare patient population, data from projects such as this can provide clarity in patient care. We hope this study prompts future investigations into further refinement of the management for patients with NMACA.

DISCLOSURE There are no conflicts of interest.

\section{REFERENCES}

1. Glasgow SC, Gaertner W, Stewart D, et al. Clinical practice guidelines for the management of appendiceal neoplasms. The American Society of Colon and Rectal Surgeons. Dis Colon Rectum. 2019;62:1425-38.

2. AlMasri SS, Hammad AY, Singhi AD, et al. Appendectomy is oncologically equivalent to right hemicolectomy for well-differentiated T1 appendiceal adenocarcinoma. Dis Colon Rectum. 2021. https://doi.org/10.1097/DCR.0000000000002089.

3. Straker RG, Sharon C, Shannon A, Faker D, Shanmugan S, Miura J, Karakousis G. Pathologic factors assocaited with low risk of lymph node metastasis in non-mucinous adenocarcinoma of the appendix. Ann Surg Oncol. 2021. https://doi.org/10.1245/s10434021-11213-5.

4. Wang G, Li Q, Chen W. Chemotherapy in the treatment of different histological types of appendiceal cancers: a SEER based study. BMC Cancer. 2021;21:778.

5. AlMasri SS, Paniccia A, Hammad AY, et al. The role of adjuvant chemotherapy following right hemicolectomy for non-metastatic mucinous and nonmucinous appendiceal adenocarcinoma. J Gastrointest Surg. 2021. https://doi.org/10.1007/s11605-021-05076-0

6. The Chicago Consensus on Peritoneal Surface Malignancies. management of appendiceal neoplasms. Ann Surg Oncol. 2020;27:1753-60.

Publisher's Note Springer Nature remains neutral with regard to jurisdictional claims in published maps and institutional affiliations. 